\title{
VERIFICATION OF SELECTED MODELS OF THE SIZE EFFECT BASED ON HIGH-CYCLE FATIGUE TESTING ON MINI SPECIMENS MADE OF EN AW-6063 ALUMINUM ALLOY
}

\author{
Tomasz Tomaszewski, Janusz Sempruch, Tomasz Piątkowski \\ University of Technology and Life Sciences in Bydgoszcz, Faculty of Mechanical Engineering, Bydgoszcz, Poland \\ e-mail: tomaszewski@utp.edu.pl
}

\begin{abstract}
The study presents several cases where use of specimens with reduced overall dimensions, i.e. mini specimens in the course of fatigue tests is advantageous. The high-cycle fatigue tests based on a developed method have been performed on mini specimens and normative specimens (comparative tests). The tests have been conducted on EN AW-6063 aluminum alloy specimens. A correction coefficient determined as a result of monotonic tests and selected models of the size effect have been critically assessed based on the test results. A statistical Weibull's weakest link model and a monofractal approach based on a fractal dimension have been verified.
\end{abstract}

Keywords: mini specimen, high-cycle fatigue, weakest link theory, fractal approach

\section{Introduction}

Taking the size effect into consideration in the engineering practice is a known issue. It does not allow using the material properties from normative tests for specimens with a different cross-sectional area. The issue applies to both specimens with smaller and larger dimensions compared to the normative specimen.

A size effect coefficient (cross-section size $K$ ) may be determined based on the durability tests on specimens with different dimensions. The coefficient value is expressed as follows

$$
K_{Z}=\frac{Z}{Z_{n}} \quad K_{H C}=\frac{\sigma}{\sigma_{n}} \quad K_{S}=\frac{R_{m}}{R_{m n}}
$$

where $Z / \sigma / R_{m}$ is the fatigue limit/fatigue strength/ultimate tensile strength of a specimen with any cross-section, $Z_{n} / \sigma_{n} / R_{m n}$ is the fatigue limit/fatigue strength/ultimate tensile strength of the normative specimen (cross-section $20-80 \mathrm{~mm}^{2}$ ) prepared using the identical material.

The size effect is defined based on probabilistic models. The probability of material defects resulting in fatigue crack increases with the specimen volume (Kocańda and Szala, 1997). The effect was proven in the experimental studies on small specimens (Furuya, 2011; Haftirman, 2009; Hirose et al., 2000) confirming that metals are sensitive to changes in the cross-section. Figure 1 shows the size effect test results for aluminum alloys.

In general, the size effect reduces fatigue strength and fatigue life with an increase in the specimen size. The results show a linear change of the coefficient $K$ (in a specific dimension range) in relation to the cross-sectional area of the specimen. The impact of the size effect on mechanical properties depends on the type and local features of the material structure (grain size, microcracks, inclusions, discontinuities, dislocations and other defects). It is particularly significant for large objects, e.g. bridge components, concrete slabs, hulls, bulkheads, decks and masts.

Strength testing of structural materials (monotonic, fatigue) is mostly conducted on normative specimens (PN-74/H-04327) with a repeatable quality at specific geometry and dimensions. 


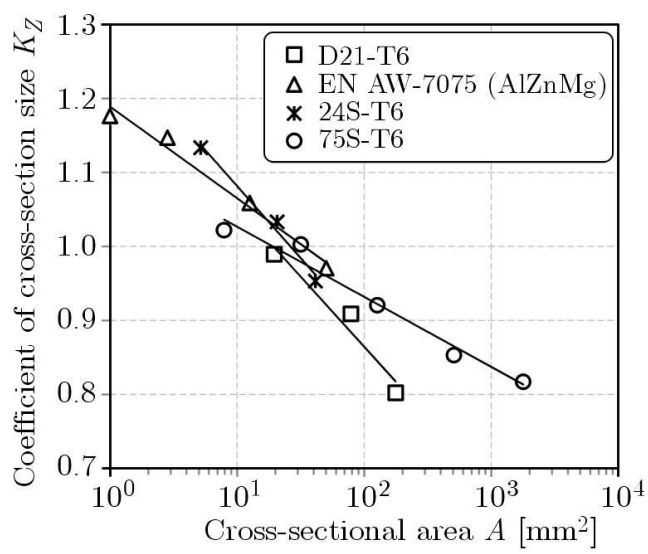

Fig. 1. Relation between the coefficient $K_{Z}$ to cross-sectional area of round, bent specimens; own data based on (Brueggeman and Mayer, 1948; Hyler et al., 1954; Troshchenko and Sosnowski, 1987)

The tests on real-life objects (in operation) are limited (material and instrumentation availability, testing costs). The tests on smaller specimens (mini specimens) are dedicated for specific conditions depending on the object and the nature of the test, which translates directly into availability of the test results for non-standard specimens. The trend is shown in Fig. 2 as a quantitative distribution of the relation between the probability of obtaining experimental data and the cross-sectional area of the specimen.

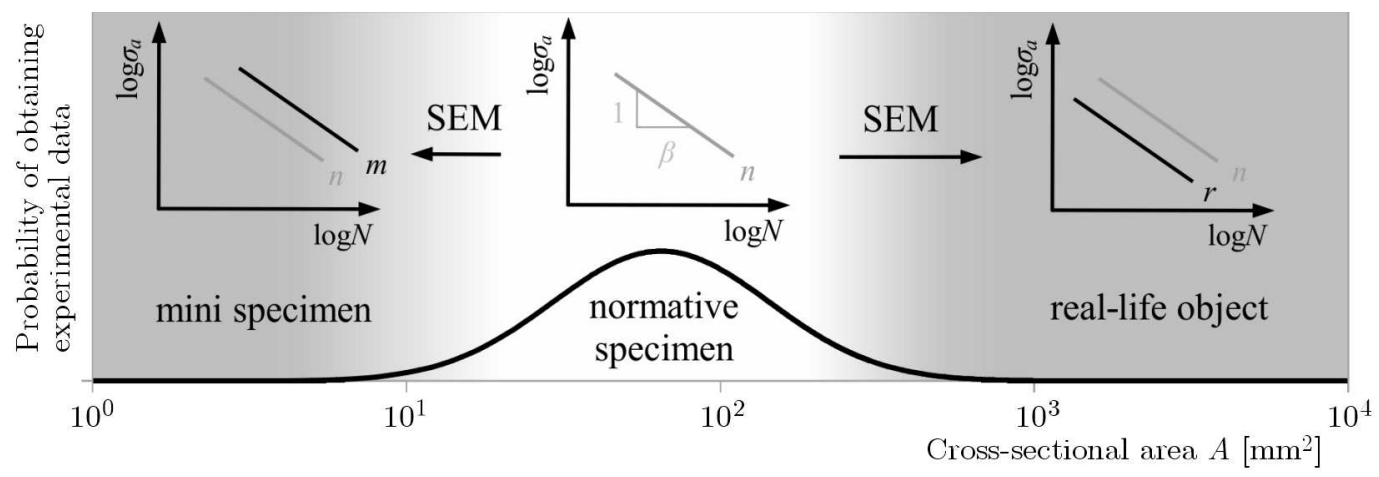

Fig. 2. Schematic approach to quantitative distribution of the probability of obtaining experimental data for different cross-sectional areas

The following mathematical models of the size effect (SEM) have been presented to estimate the normative test results (laboratory) to specimens with different geometry (cross-sectional area). One of the first models is a statistical model based on the weakest link theory (Weibull, 1939). Another concept is a fractal approach with a fractal dimension depending on the material structure (Carpinteri et al., 2001, 2009). The literature also contains references to the size effect law (Bažant, 1984) and a critical volume model (Sosnovski, 1990).

The models are used for objects with the similar geometry and made of the identical material. The models may be used to estimate fatigue characteristics for specimens with different cross-sectional areas. Figure 3 shows $\sigma_{a}-N$ curves for specimens with a cross-section $A_{A}$ and $A_{B}$ subject to a one-axis load.

The discussed approach to the size effect issue allows one to use the presented models to estimate the high-cycle fatigue performance of specimens with lower or higher cross-sectional area than the normative specimens. This approach is checked if mini specimen tests are appropriate.

A test method based on mini specimens has been suggested due to the extension of the scope of fatigue tests outside the standard guidelines (imposed specimen geometry). It applies to the 


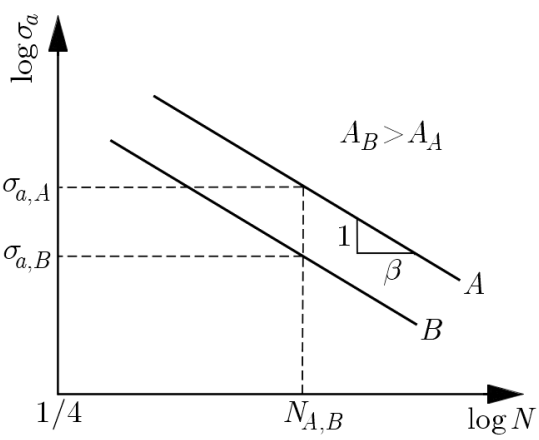

Fig. 3. $\sigma_{a}-N$ curves in a double-logarithmic coordinate system for specimens with different cross-sectional areas

(a) mini specimen

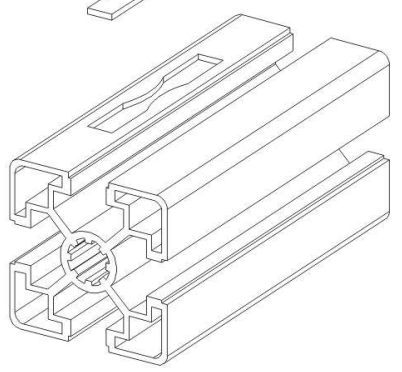

(b)

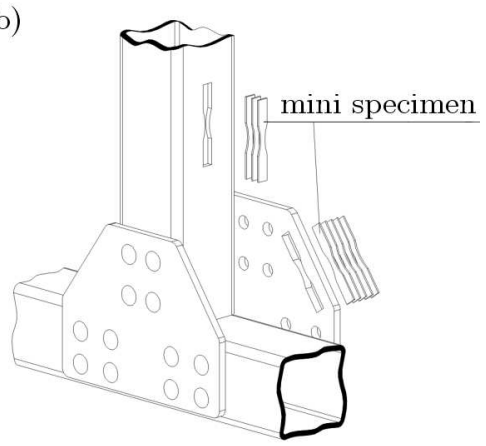

(c)

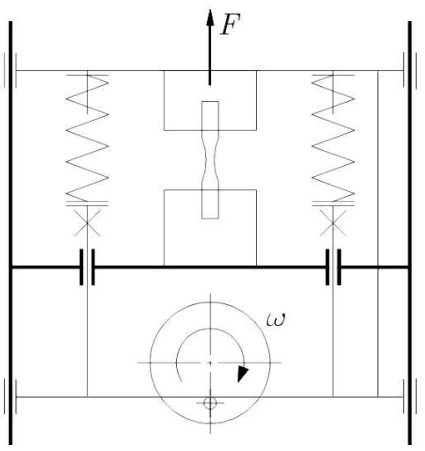

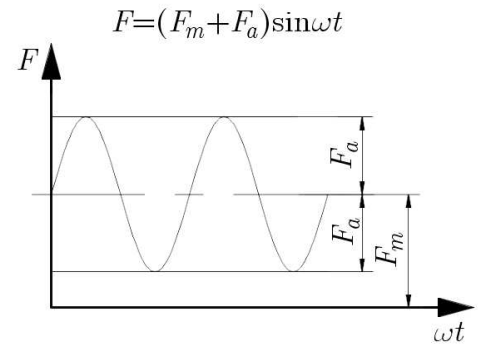

Fig. 4. Examples of mini specimen application: (a) sampling from a thin-walled structure, (b) sampling from a component in operation, (c) test on a test stand with simplified loading system

cases where sampling is limited due to the size of a structural object. The tendency to dimension the machine components by rigidity (thin, geometrically developed walls) and not the strength leads to manufacturing of thin-walled objects with complex shapes. The examples include profile sections (Fig. 4a) made of an aluminum alloy used in the extrusion moulding process. Strength and cyclic properties of a pre-cast material change as a result of plastic strain specific to the process. The actual fatigue life can be determined by testing the specimens sampled from the finished components only.

Another approach is to verify the mechanical properties of the material of a structural element in operation to determine the failure rate (damage) compared to a new component. The examples include the structural nodes showed in Fig. 4b. A significantly lower volume of the mini specimens allows comprehensive fatigue test even with a limited availability of the source material. The impact of interference on the construction is greatly reduced.

The determination of fatigue performance is in many cases limited due to financial considerations (availability of testing machines, test lead time etc.). A range of used loads may be reduced to lower the costs. Also smaller forces are achievable at the test stand for reduced cross- 
-sectional areas of the specimens. It allows using simplified loading systems, e.g. with a kinematic excitation (Fig. 4c) to reduce the operating and test stand investment costs.

This study aims to at verification of the size effect models within the scope of the results of the experimental tests on non-standard and normative specimens. The non-standard specimen is a specimen geometrically smaller (mini specimen) from the normative specimen. A criterion to select the model is a quality of matching the estimated fatigue life of the normative specimen based on the base performance data (obtained for a non-standard specimen).

\section{Mathematical size effect models}

\subsection{Weakest link theory (Weibull, 1939)}

A statistical model of the weakest link theory is based on the Weibull probability distribution of failure. The theory describes a scatter of strength properties of brittle materials (e.g. ceramics). It is also used for testing cyclic loading of metals (Weibull, 1949). It results from the nature of the failure itself. A fatigue crack is initiated by a randomly distributed material defects as in the case of brittle materials. The theory is used in high-cycle fatigue tests to determine a fatigue life allowing for the stress gradient effect (Karolczuk and Palin-Luc, 2013) or to define the scatter of strength properties in multiaxial fatigue (Flaceliere and Morel, 2004).

The theory is based on statistically distributed defects (non-homogeneities, inclusions, cracks, precipitates) in a material per volume unit. A crack is initiated in a specific volume where the most dangerous defect or the weakest link exists. The propagation of fatigue cracks is independent in different areas. The Weibull distribution relates the effect of load and cross-sectional area with the fatigue life. It may be used in a qualitative determination of the effect of cross-sectional area. A classic form of the Weibull distribution for the failure probability is

$$
P(\sigma, \Omega)=1-\exp \left[-\frac{1}{\Omega_{o}} \int_{\Omega} f(\sigma) d \Omega\right]
$$

where $\Omega_{o}$ is the reference volume or surface, $f(\sigma)$ is a function of the risk of rupture (two or three parameters)

$$
f(\sigma)=\left(\frac{\sigma}{\sigma_{o}}\right)^{m} \quad f(\sigma)=\left(\frac{\sigma-\sigma_{u}}{\sigma_{o}}\right)^{m}
$$

where $m$ is the shape parameter of the Weibull distribution function, $\sigma_{u}$ is the threshold stress, $\sigma_{o}$ is the scale parameter.

The results of high-cycle fatigue tests are defined with a cumulative distribution function of failure probability (two parameters) of a function depending on the number of cycles $(P=f(N))$. The fatigue life for a specific stress level shows a certain scatter with the distribution defined on a logarithmic scale (Schijve, 2009) and is expressed as follows

$$
P(N)=1-\exp \left[-\left(\frac{\log N}{\log N_{o}}\right)^{m}\right]
$$

where $N_{o}$ is the reference fatigue life (probability 0.63) for a specific stress level, $m$ is the distribution shape coefficient corresponding to the fatigue life scatter bandwidth $N$.

If the distribution of the results for the tested material may be expressed by Eq. (2.1), the results may be estimated for specimens with different cross-sectional areas. The distribution shape parameter $m$ and the reference fatigue life $N_{o}$ are material constants independent of the 
specimen size and stress distribution. The following relation may be used for a similar failure probability, stress distribution and two different specimen sizes

$$
\frac{N_{2}}{N_{1}}=\left(\frac{A_{1}}{A_{2}}\right)^{\frac{1}{m}}
$$

where $N_{1}$ is the fatigue life for a specimen with the known surface (cross-sectional area) $A_{1}, N_{2}$ is the estimated fatigue life for the specimen with determined surface (cross-sectional area) $A_{2}$. The failure probability value based on experimental data is estimated as follows

$$
p=\frac{i-0.5}{n}
$$

where $i$ is the ordinal test number $(1,2, \ldots, n), n$ is the number of tested specimens for a specific load level.

\subsection{Model based on the one-dimension fractal approach (Carpinteri et al., 2001, 2009)}

A size effect is described using a fractal approach, where the material structure ligaments are modelled using a fractal set (as in the case of Cantor's mathematical set). The crack area is modelled using similar fractal sets with geometry based on the von Koch curve. New mechanical properties may be determined based on physical dimensions depending on the fractal dimension treated as the fractional dimension. The material ligament failures or the macroscopic cracks are modelled using this model.

The basic law of linear scaling of fractals is a one-dimension fractal approach (monofractal - nomenclature as per Carpinteri et al. (2009)) using the fractal dimension. A non-linear model was presented (multifractal approach), where the material properties were defined by three parameters. It applies to large objects, where the effect of micro-structure and micro-failures on mechanical properties is gradually reduced with an increase in object dimensions.

The monofractal approach can be used to determine the size effect on material fatigue. It is based on Basquin's equation to define the results of the experimental high-cycle fatigue tests

$$
C=N\left(\sigma_{a}\right)^{\beta}
$$

where $C$ is a constant parameter, $\beta$ is the slope of the $\sigma_{a^{-}} N$ curve. Assuming the parallelism ( $\beta=$ const $)$ between the $\sigma_{a}-N$ curves for specimens with different size and the relation between the constant $C$ parameter and the cross-sectional area $A$, the following may be derived

$$
C_{B}=C_{A}\left(\frac{A_{A}}{A_{B}}\right)^{-d \beta}
$$

where $A_{A}, A_{B}$ are the cross-sectional areas for geometrically similar specimens $A$ and $B$ assuming that $A_{B}>A_{A}, C_{A}, C_{B}$ are constant parameters of the $\sigma_{a^{-}} N$ curve, $d \beta$ is the slope of the curve (Fig. 5). The relation between the cross-sectional area and the material structure is described by the fractal dimension $\alpha=2-d(0 \leqslant d \leqslant 0.5)$. For $A_{A}=1, A_{B}=A$ and $C_{A}=C_{1}$ for the cross-sectional area $A=1$, Eq. (2.7) it is expressed as

$$
C(A)=C_{1}(A)^{-d \beta}
$$

The position of the $C(A)-A$ curve is determined based on constant parameters $C$ of the $\sigma_{a}-N$ curves for specimens with different cross-sectional areas $A$. $C(A)$ tend towards zero for specific characteristics. Thus $\sigma_{a}$ tends towards zero for each $N$ value. Therefore, the monofractal approach is true for a narrow range of object dimensions. Equations (2.7), (2.8) define the sensitivity of a material or a group of materials to the change in the cross-sectional area. 


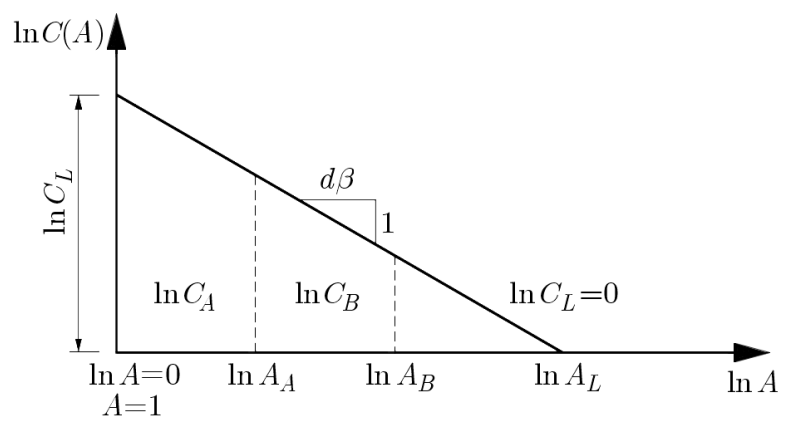

Fig. 5. Relation between the $C(A)$ parameter of the $\sigma_{a}-N$ curve and the cross-sectional area $A$ in a bilogarithmic scale

\subsection{Other models}

A model presented by Bažant (1984) is a deviation from the standard size effect theory. The total potential energy release (caused by a fatigue crack) depends on the crack length and propagation area. The size of the crack propagation area is constant and independent of the structure dimensions.

A statistical model of the size effect presented by Sosnovski (1990) assumes a limited area (finite dimensions) with a critical level of normal stresses within the specimen. The area is known as the critical volume of solid body deformation. It depends on volume, geometry, stress gradient and distribution function parameters for a specific probability. A statistical solid body includes a set of basic elements, each to a degree responsible for the strength of the entire body. The critical volume of the specimen is defined as 'a measure of responsibility' of each elementary volume.

\section{Experimental tests}

\subsection{Test conditions}

High-cycle fatigue tests have been performed on specimens with two different geometries (normative specimen (PN-74/H-04327), mini specimen - Table 1) of similar shape. The specimens were made of EN AW-6063 aluminum alloy. The tested materials were taken as flat bars with the same thickness as the specimens to avoid machining. The specimen geometry was imposed by an aspect ratio $\alpha_{k}$ of 1.05. The mini specimens were machined in sets (milling). The surface was not ground or polished.

Table 1. Specimen dimensions

\begin{tabular}{|l|c|c|c|c|}
\hline & $\begin{array}{c}\text { Thickness } \\
{[\mathrm{mm}]}\end{array}$ & $\begin{array}{c}\text { Min. width } \\
{[\mathrm{mm}]}\end{array}$ & $\begin{array}{c}\text { Radius } \\
{[\mathrm{mm}]}\end{array}$ & $\begin{array}{c}\text { Cross-sectional area } \\
{\left[\mathrm{mm}^{2}\right]}\end{array}$ \\
\hline \hline Normative specimen & 4 & 7 & 50 & 28 \\
\hline Mini specimen & 1 & 3.5 & 25 & 3.5 \\
\hline
\end{tabular}

The experimental tests were conducted for the high-cycle fatigue with controlled stress. A macro crack was the test end criterion. The aluminum alloys showed cyclic hardening above the yield point. It was observed in the course of cyclic tests on prestrain specimens, where a $15 \%$ increase in yield point was reported (Tomaszewski and Sempruch, 2012). A 190 MPa (normative specimen) and $210 \mathrm{MPa}$ (mini specimen) stress was taken as the upper limit of high-cycle fatigue. A fatigue life of $1 \cdot 10^{6}-5 \cdot 10^{6}$ cycles was taken as the lower limit of the tested area (Sonsino, 2007). The tests were performed at a $5 \mathrm{~Hz}$ frequency of the load change. A load cycle with a stress ratio $R=0.1$ which eliminated the mini specimen buckling was used. The tests were performed on the servo-hydraulic testing machine Instron 8874. 


\subsection{Test results in monotonic load conditions}

The mechanical properties of the material were measured via a static tensile test compliant with the norm (PN-EN ISO 6892-1:2010). The test was performed on specimens with a cross-sectional area corresponding to the specimens used in fatigue tests. The properties were determined for two geometries (five specimens each). Table 2 and Fig. 6 show the average results. The results indicate a relation between the strength properties and the cross-sectional area.

Table 2. Monotonic material properties

\begin{tabular}{|c|c|c|c|}
\hline$R_{m}[\mathrm{MPa}]$ & $R_{e}[\mathrm{MPa}]$ & $A[\%]$ & $Z[\%]$ \\
\hline \hline \multicolumn{2}{|c|}{ Normative specimen $\left(A=28 \mathrm{~mm}^{2}\right)$} \\
\hline 200 & 167 & 16.6 & 61.2 \\
\hline \multicolumn{4}{|c|}{ Mini specimen $\left(A=3.5 \mathrm{~mm}^{2}\right)$} \\
\hline 230 & 208 & 10.8 & 53.0 \\
\hline \multicolumn{4}{|c|}{$K_{S}$} \\
\hline 1.15 & 1.25 & - & - \\
\hline
\end{tabular}

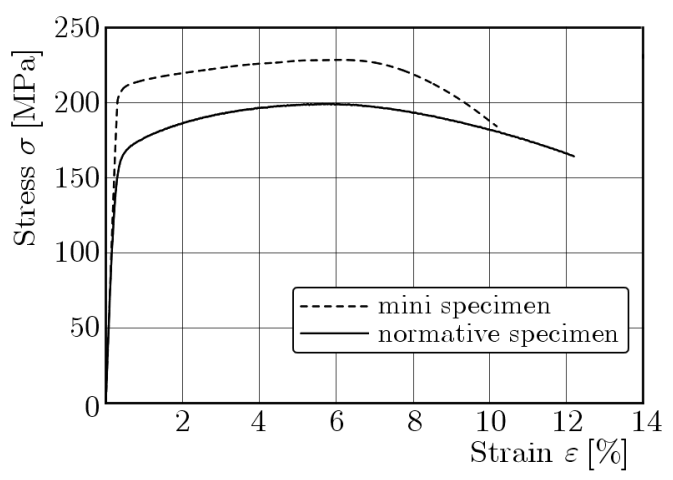

Fig. 6. Static tensile plot

\subsection{Test results in fatigue load conditions}

Figure 7 shows the fatigue test results. The experimental points were approximated to the linear form $\log \sigma_{a}=a \log N+b$, for which the confidence interval of 0.95 was determined.

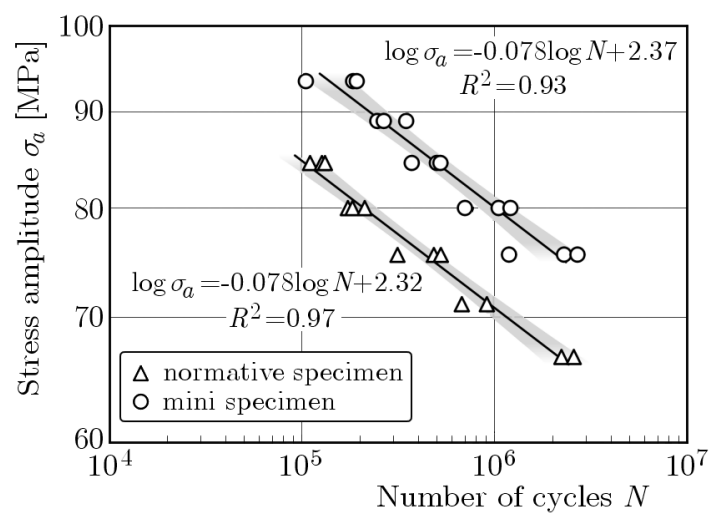

Fig. 7. $\sigma_{a}-N$ curves for the normative and mini specimen

The correlation coefficient $R^{2}(0.93$ - mini specimen, 0.97 - normative specimen) was used as a measure of matching the regression model. High values reflect quality and repeatability 
of the results. Despite high agreement between the slope coefficient $a$ of the regression line, the test of significance was performed. There were no premises to discard the hypothesis on the equality of the slope coefficients $a$ (parallel in relation to $\sigma_{a}-N$ curves). The scatter of strength properties for the tested specimens was described with a coefficient $K_{H C}$ independent of the stress level. The position of specimen crack with a random distribution was measured. It indicated no effect of the geometry shape, machining and other factors on the test results (Tomaszewski and Sempruch, 2014).

\section{Implementation of the size effect models}

The selected models of the size effect theory were used to estimate the fatigue life of the normative specimen. The fatigue life for the cross-sectional area of this specimen was determined using the $\sigma_{a}-N$ curve received for the mini specimen. The regression line obtained as a result of the experimental tests on the normative specimens was used as the reference. The coefficient of residual variation and the standard error of the estimate were used to define the matching rate of the estimated lines (based on the models) and the experimental data.

The weakest link theory was the first implemented model. The estimated failure probability (2.5) was non-linear approximated to a cumulative distribution function of the two-dimensional Weibull distribution as in Eq. (2.3). The reference fatigue life $N_{o}$ and $m$ parameter values were determined for the stress amplitude $\sigma_{a}$ (Table 3). Figure 8 shows experimental points on the $\sigma_{a}-N$ curve with the fatigue life scatter for $P=0.05,0.63,0.95$. The fatigue life for the normative specimen was estimated based on Eq. (2.4).

Table 3. Probability distribution model parameters resulting from Eq. (2.3) for the results of the experimental tests on the mini specimen

\begin{tabular}{|c|c|c|}
\hline$\sigma_{a}[\mathrm{MPa}]$ & $\log N_{o}$ & $m$ \\
\hline \hline 93.6 & 5.261 & 40.01 \\
\hline 89 & 5.491 & 65.49 \\
\hline 84.6 & 5.704 & 85.08 \\
\hline 80 & 6.040 & 61.92 \\
\hline 75.6 & 6.374 & 42.39 \\
\hline
\end{tabular}

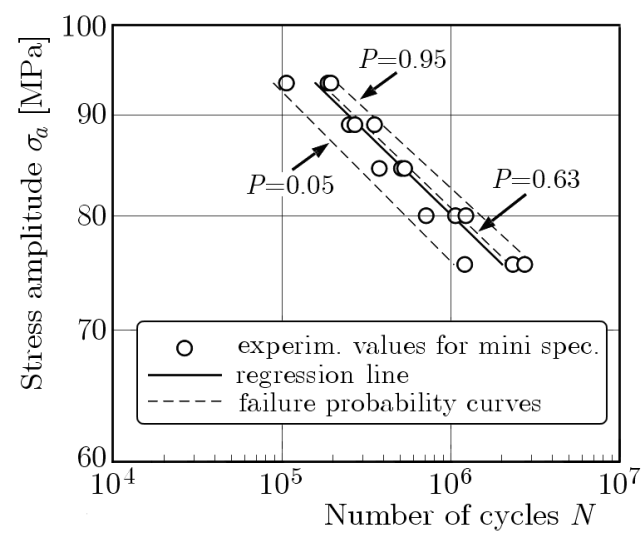

Fig. 8. $\sigma_{a}-N$ curve with experimental values and fatigue life scatter for $P=0.05,0.95$ and the reference fatigue life $(P=0.63)$ of the Weibull distribution

The monofractal approach was also applied due to the extent of tested geometry sizes. The basic characteristic (mini specimen) was defined using Eq. (2.6) as $2.0013 \cdot 10^{30}=N\left(\sigma_{a}\right)^{12.76}$. 
The fractal dimension for aluminum alloys is $\alpha=1.934, d=0.066$ (based on the analysis of four aluminum alloys in Tomaszewski and Sempruch (2013)). Based on the results of the experimental tests on the mini specimen, curve Eq. (2.7) was plotted (relation between the constant parameter $C$ and the specimen cross-sectional area $A$ on a natural logarithmic scale). The constant $C_{B}$ is calculated from Eq. (2.7) for a defined cross-sectional area $\left(A_{B}\right)$ of the normative specimen. Figure 9 shows the experimental values (mini specimen) and the estimated values (normative specimen) along the $C$ - $A$ curve. This approach allowed one to determine values of The $C$ parameter for specimens with different cross-sectional areas while maintaining the assumed constant slope of the $\sigma_{a^{-}} N$ curves.

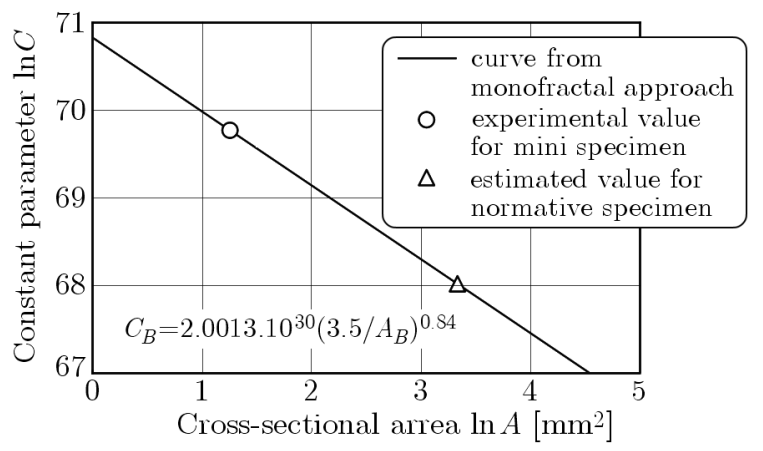

Fig. 9. Relation between the parameter $C$ and cross-sectional area $A$ for EN AW-6063 aluminum alloy

\section{Results analysis}

The results of the experimental tests and applied models of the size effect were defined with a linear regression model with a slope coefficient $a$ and an absolute term $b$ (Table 4).

Table 4. Parameters of the inear regression model, standard error of the estimate and the coefficient of variation in relation to empirical values

\begin{tabular}{|c|c|c|c|c|}
\hline \multirow{3}{*}{ Data } & \multirow{2}{*}{\multicolumn{2}{|c|}{ 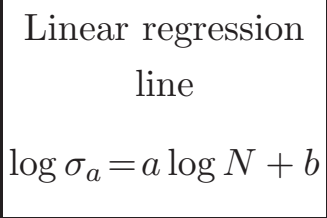 }} & \multirow{3}{*}{$\frac{S_{e}=\sqrt{\frac{1}{n-2} \sum_{i=1}^{n}\left(y_{i}-y_{i e s t}\right)^{2}}}{S_{e}[\text { cycles }]}$} & \multirow{3}{*}{$\begin{array}{c}\begin{array}{c}\text { Coefficient of } \\
\text { residual variation } \\
V_{e}=\frac{S_{e}}{\bar{y}} \cdot 100 \% \\
V_{e}[\%]\end{array} \\
\end{array}$} \\
\hline & & & & \\
\hline & $a$ & $b$ & & \\
\hline $\begin{array}{l}\text { Mini specimen } \\
\text { experimental tests }\end{array}$ & -0.078 & 2.37 & 331518 & 41.3 \\
\hline $\begin{array}{l}\text { Normative specimen } \\
\text { experimental tests }\end{array}$ & -0.078 & 2.32 & 162209 & 24.1 \\
\hline Weakest link theory & -0.088 & 2.41 & 930951 & 138.1 \\
\hline Monofractal approach & -0.078 & 2.33 & 285788 & 42.4 \\
\hline $\begin{array}{l}\text { Coefficient } K_{S} \text { for } R_{m} \\
\text { from monotonic test }\end{array}$ & -0.078 & 2.31 & 309815 & 45.9 \\
\hline
\end{tabular}

Figure 10e shows the results of the experimental test (normative specimen - confidence interval, mini specimen - regression line) and dashed lines showing the fatigue life results based on the models (item 2 - weakest link model Eq. (2.4), monofractal approach Eq. (2.7)) and the dotted line (based on the coefficient $K_{S}$ resulting from the monotonic tests). The curves were calculated based on the results of the experimental test on mini specimens. The equivalent 
fatigue life values were determined for the normative specimen. Figure 10e shows the analysed models (allowing for the size effect) result in the offset of $\sigma_{a}-N$ curves in the right direction.

Figure 10a shows the fatigue life scatter (experimental tests) for specimens with different geometry. The confidence interval of the results of the tests on the normative specimens does not correspond to the results obtained for the mini specimens. It is due to evident impact of the size effect on fatigue life of the specimens made of EN AW-6063 aluminum alloy. Its value is defined with the coefficient $K_{H C}$ with a mean value of the stress amplitude of 1.13.

The coefficient $K_{S}$ resulting from Eq. (1.1) 3 was used to estimate the fatigue life based on monotonic tests. Figure 10b shows that in specific conditions, the coefficient $K_{S}$ may be used as a correction coefficient for the parallel characteristics offset (for mini specimen) in the direction of lower fatigue life values. The high satisfaction level resulting from the quality of the results requires further verification for different groups of structural materials.

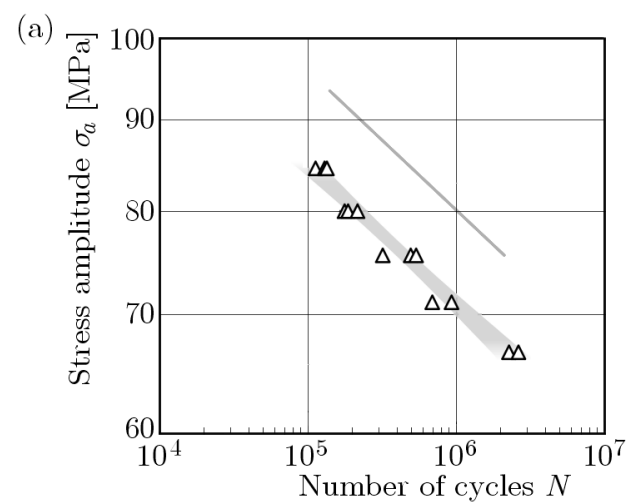

(c)

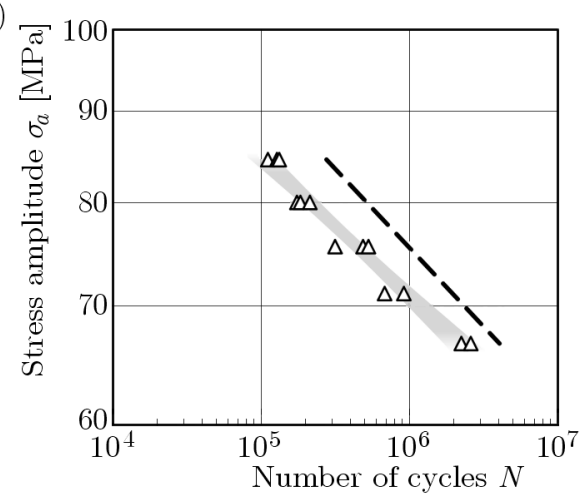

(e)

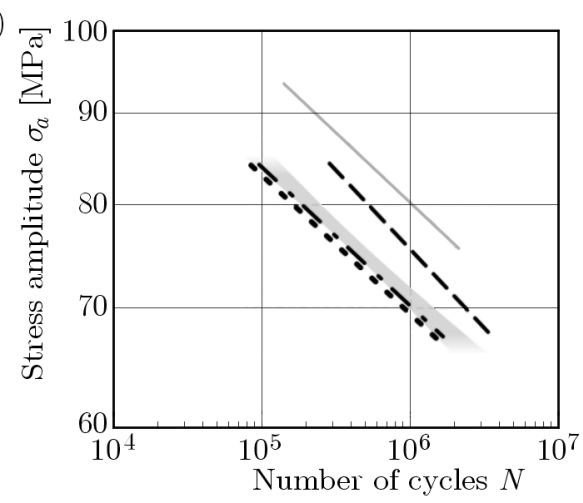

(b)

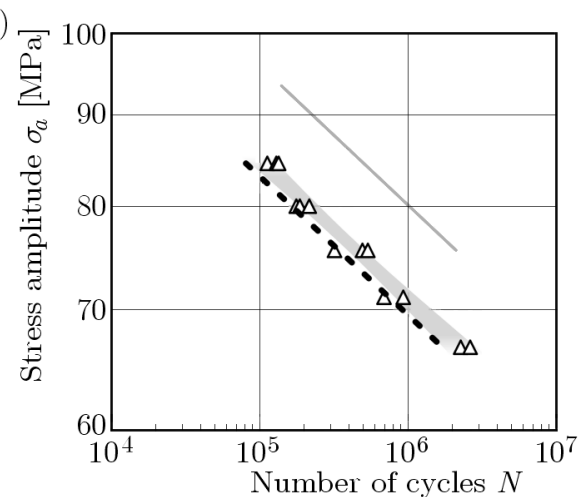

(d)

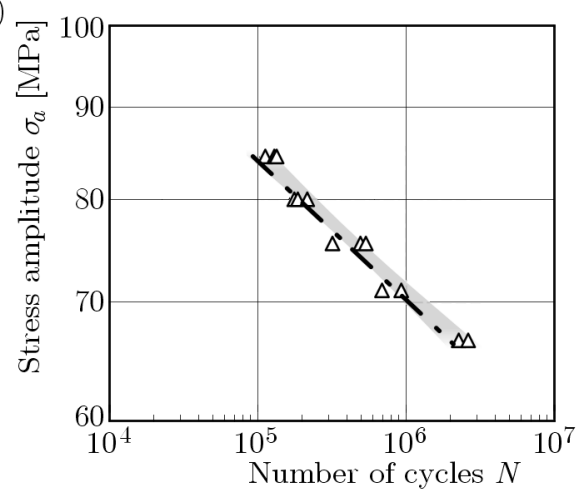

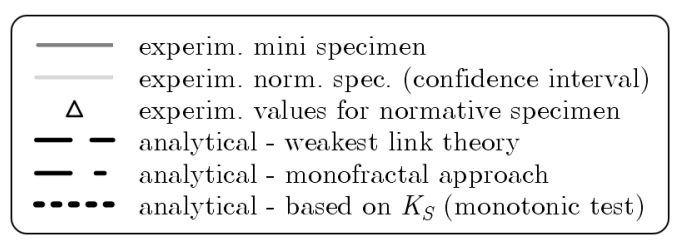

Fig. 10. Fatigue curves plotted based on the experimental tests and mathematical models: (a) mini specimen (experimental), normative specimen (experimental), (b) normative specimen (experimental), monotonic tests (analytical - based on $K_{S}$ ), (c) normative specimen (experimental), weakest link theory (analytical), (d) normative specimen (experimental), monofractal approach (analytical), (e) comparison $\sigma_{a}-N$ curves 
Figures 10c and 10d show that the better approximation (compared the weakest link model) was obtained using the monofractal model with the coefficient of residual variation equal to $42.4 \%$. The fractal model locates the $\sigma_{a}-N$ curve on the safe side. The estimated fatigue life values are lower than the regression line for experimental points.

\section{Conclusions}

The intention of the study is to recall the necessity of taking into consideration the size effect in structural calculations. Some inaccuracies found in calculations using various fatigue strength and fatigue life models in mechanical engineering are a result of neglecting the size effect.

The experimental tests show a significant impact of the size effect on both monotonic and fatigue loads. Its value is defined by a size effect coefficient of $1.15\left(K_{S}\right)$ and $1.13\left(K_{H C}\right)$. Despite the diverse nature of specimen failure in monotonic and fatigue tests, high conformity of the size effect coefficient is observed.

The weakest link theory model which only requires the Weibull distribution parameters for the $\sigma_{a}-N$ curve is the least complex among the verified theoretical models. The monofractal approach requires a mean value of the fractal dimension based on the results of the experimental tests on the size effects available in the literature (at least two different cross-sectional areas) for a group of materials (in this case four different aluminum alloys).

The applied models allow an offset of the basic characteristics in the right direction (Fig. 10e) to estimate the fatigue life with lower values. It is consistent with the experimental results. Weibull's model does not reflect the material constants and thus the results are affected by a $138.1 \%$ error. The $\sigma_{a}-N$ curve is characterised by a different slope inconsistent with the results of the experimental tests. An accurate approximation is possible with the monofractal model affected by a $42.4 \%$ error.

The characteristics offset using the $K_{S}$ coefficient is determined by an unexpectedly low error $(45.9 \%)$ considering the divergence in the definition of monotonic and fatigue tests. This conclusion shall be treated as a signal rather than an approval of the applicability of this value.

\section{References}

1. BAŽAnt Z.P., 1984, Size effect in blunt fracture concrete, rock, metal, Journal of Engineering Mechanics ASCE, 110, 518-535

2. Brueggeman W.C., Mayer M.JR., 1948, Axial fatigue tests at zero mean stress of 24S-T and 75S-T aluminum-alloy strips with a central circular hole, National Advisory Committee for Aeronautics, 1611, 1-24

3. Carpinteri A., Spagnoli A., Vantadori S., 2001, An approach to size effect in fatigue of metals using fractal theories, Fatigue and Fracture of Engineering Materials and Structures, 25, 619-627

4. Carpinteri A., Spagnoli A., Vantadori S., 2009, Size effect in S-N curves: A fractal approach to finite-life fatigue strength, International Journal of Fatigue, 31, 927-933

5. Flaceliere L., Morel F., 2004, Probabilistic approach in high-cycle multiaxial fatigue: volume and surface effects, Fatigue and Fracture of Engineering Materials and Structures, 27, 1123-1135

6. Furuya Y., 2011, Notable size effects on very high cycle fatigue properties of high-strength steel, Materials Science and Engineering A, 528, 5234-5240

7. Haftirman, 2009, The size effect on fatigue strength of structural steel materials in high-humidity environment, Proceedings of International Conference on Applications and Design in Mechanical Engineering 
8. Hirose T., Sakasegawa H., Kohyama A., Katoh Y., Tanigawa H., 2000, Effect of specimen size on fatigue properties of reduced activation ferritic/martensitic steels, Journal of Nuclear Materials, 283/287, 1018-1022

9. Hyler W.S., Lewis R.A., Groverh J., 1954, Experimental investigation of notch-size effects on rotating-beam fatigue behaviour of 75S-T6 aluminum alloy, National Advisory Committee for Aeronautics, 3291, 1-47

10. Karolczuk A., Palin-Luc T., 2013, Modelling of stress gradient effect on fatigue life using Weibull based distribution function, Journal of Theoretical and Applied Mechanics, 51, 297-311

11. Kocańda S., Szala J., 1997, Bases of Fatigue Calculation (in Polish), PWN

12. PN-74/H-04327 The study of metal fatigue. The test of axial tension-compression at constant cycle of external loads (in Polish)

13. PN-EN ISO 6892-1:2010 Metals - Tensile testing - Part 1: Test method at room temperature (in Polish)

14. Schijve J., 2009, Fatigue of Structures and Materials, Springer

15. Sonsino C.M., 2007, Course of SN-curves especially in the high-cycle fatigue regime with regard to component design and safety, International Journal of Fatigue, 29, 2246-2258

16. Sosnovski L.A., 1990, Statistical model of a deformable solid with a critical volume and some of its applications, Strength of Materials, 22, 626-632

17. Tomaszewski T., Sempruch J., 2012, Determination of the fatigue properties of aluminum alloy using mini specimen, Materials Science Forum, 726, 63-68

18. Tomaszewski T., Sempruch J., 2013, Application of monofractal approach to describe size effect in fatigue life prediction for aluminum alloys, Materials of 30th Danubia-Adria Symposium, 173-174

19. Tomaszewski T., Sempruch J., 2014, Verification of the fatigue test method applied with the use of mini specimen, Key Engineering Materials, 598, 243-248

20. Troshchenko V.T., Sosnowski L.A., 1987, Fatigue Resistance of Metals and Alloys (in Russian), Academy of Sciences of the Ukrainian SSR

21. Weibull W., 1939, A statistical theory of the strength of materials, Royal Swedish Institute For Engineering Research, 151

22. Weibull W., 1949, A statistical representation of fatigue failures in solids, Transaction of the Royal Institute of Technology, $\mathbf{2 7}$ 